\title{
Association of Ferrara Intracorneal Ring Segments with Phakic Intraocular Lens for Improved Visual Acuity in Young Keratoconus Patients: A Case Series
}

\author{
Edna M Almodin ${ }^{1}$, Flávia Almodin², Juliana Almodin ${ }^{3}$
}

\begin{abstract}
Aim: We present a case series involving five eyes in four young patients with advanced keratoconus who were implanted with Ferrara intrastromal corneal ring segments (ICRS) followed by phakic intraocular lens (PIOLs).

Background: ICRS have been used for the management of keratoconus in order to prevent further keratoconus progression and improve visual performance. Nonetheless, many patients may still suffer from moderate to high myopia after ICRS, requiring refractive error correction, which may not be achieved with glasses, contact lenses, or laser refractive surgery. For such cases, PIOLs have emerged as an alternative to correct residual refractive errors.

Case description: Patients were aged between 13 years and 32 years at ICRS implantation. Around 6 months after ICRS implantation, patients were submitted to a second procedure for the implantation of an implantable collamer lens (ICL), Artisan or Artiflex PIOL to correct residual myopia. After the combined Ferrara ICRS and PIOL implantation, all treated eyes demonstrated a significant improvement in topographic findings and visual acuity, with reduced refraction and keratometric astigmatism.

Conclusion: The combined procedure was successful in reshaping the cornea and improving visual acuity of patients.

Clinical significance: The association of ICRS and PIOL can be an important alternative that should be considered to provide young patients with improved visual acuity in a moment of their lives when they are at their most productive phase.

Keywords: Intracorneal ring segments, Keratoconus, Phakic lens.

International Journal of Keratoconus and Ectatic Corneal Diseases (2019): 10.5005/jp-journals-10025-1175
\end{abstract}

\section{INTRODUCTION}

Keratoconus is a progressive disease that affects the topography of the cornea, resulting in impaired visual acuity and refractive errors. ${ }^{1,2}$ Procedures such as the implantation of intrastromal corneal ring segments (ICRS), ${ }^{3,4}$ and corneal collagen crosslinking ${ }^{5-7}$ have been recently introduced for the management of keratoconus.

After ICRS implantation, many patients may still suffer from moderate to high myopia, requiring refractive error correction. ${ }^{8}$ In cases correction may not be achieved with glasses, contact lenses, or refractive surgery with laser, ${ }^{9,10}$ phakic intraocular lens (PIOLs) emerge as a possible alternative. ${ }^{11,12}$ Previous studies with keratoconus patients with high myopia have already reported on improved visual acuity after the sequential implantation of Intacs ${ }^{8}$ and Keraring ${ }^{13}$ ICRS and implantable collamer lenses (ICLs) in the posterior chamber, and Intacs ${ }^{14}$ ICRS and Artisan in the anterior chamber.

This case series reports on the findings of a group of patients with advanced keratoconus and high myopia who were implanted with Ferrara ICRS followed by the implantation of ICL, Artisan, and Artiflex phakic intraocular lens (PIOL).

\section{Case Description}

This retrospective case series presents the results of the implantation of Ferrara ICRS followed by the implantation of ICL, Artisan, and Artiflex PIOLs in five eyes of four patients (two female and two male) with keratoconus, high myopia, and contact lens intolerance, conducted at Provisão Hospital de Olhos, Maringá,
${ }^{1-3}$ Department of Ophthalmology, Provisão Hospital de Olhos, Maringá, Brazil

Corresponding Author: Edna M Almodin, Department of Ophthalmology, Provisão Hospital de Olhos, Maringá, Brazil, Phone: +55 4432622061, e-mail: ealmodin@gmail.com

How to cite this article: Almodin EM, Almodin F, Almodin J. Association of Ferrara Intracorneal Ring Segments with Phakic Intraocular Lens for Improved Visual Acuity in Young Keratoconus Patients: A Case Series. Int J Kerat Ect Cor Dis 2019;8(1):23-28.

Source of support: Nil

Conflict of interest: None

Brazil. All the patients were systemically healthy and presented no other eye conditions.

Before and after the implantation of Ferrara ICRS and PIOL, the patients underwent a thorough eye examination, which involved uncorrected visual acuity (UCVA) and distance-corrected visual acuity (DCVA) with the Snellen chart, cycloplegic refraction, and videokeratoscopy using Tomey (Tomey Corporation, Japan) and Wavelight Oculyzer (Wavelight Allegretto, Alcon, USA).

\section{Ferrara ICRS Implantation}

All the five eyes in this case series were implanted in the steepest topographic axis of the cornea with Ferrara ICRS (AJL Ophthalmic, Valladolid, Spain) of various thicknesses and arc lengths. One or two ICRS segments were implanted depending on the topography of the cornea, whereas ICRS thickness was defined according to 
the spherical equivalent and corneal thickness. The surgery was conducted under topical anesthesia. The thickness of the cornea was obtained preoperatively using the pachymetry map (Oculyzer). The tunnel for ICRS insertion was created with a femtosecond laser equipment (Ziemer 8, Ziemer Ophthalmic Systems, Germany) at $80 \%$ of the corneal thickness at its thinnest point in the $5.0 \mathrm{~mm}$ optical zone. After the surgery, patients were instructed to apply antibiotic-steroid eye drops at every 6 hours during two weeks, avoid rubbing their eyes, and use artificial tears as required.

\section{PIOL Implantation}

PIOL implantation occurred with a minimum of 6 months after the ICRS surgery. Two of the eyes received a posterior chamber PIOL (Visian ICL; Staar Surgical, Monrovia, California, USA), two eyes were implanted with a foldable anterior chamber iris-fixated Artiflex PIOL (Ophtec, Groningen, the Netherlands), and one eye received a rigid anterior chamber iris-fixated Artisan PIOL (Ophtec, Groningen, the Netherlands). The type of lens was chosen based on the topographic astigmatism of the patient. The size of the PIOLs was defined according to the measurements of corneal white-towhite and anterior chamber depth, conducted with the loLMaster 500 (Carl Zeiss Meditec AG, Germany). Lens power calculation was performed aiming at emmetropia or slight residual myopia. All PIOLs were implanted under peribulbar anesthesia as previously described in the literature. ${ }^{8-15}$ No complications were observed either intra- or postoperatively.

- Case 1: MBS, a 19-year-old female patient. Six months after the implantation of two Ferrara ICRS (160/300) in OD, UCVA and DCVA improved significantly, and a reduction in the refraction and keratometric astigmatism was also observed. Topography and tomography showed a significant flattening of the cornea. The patient then received the implantation of an Artiflex PIOL (-9.5 D). Six months after the procedure, UCVA, DCVA, and manifest refraction were significantly improved (Fig. 1 and Table 1).

- Case 2: ELLS, 13-year-old male patient. Six months after the implantation of one Ferrara ICRS (210/200) in OD, UCVA was still count fingers but DCVA showed a significant improvement with a reduction in the refraction and keratometric astigmatism. Topography and tomography showed a significant flattening of the cornea. Six months after the implantation of an Artisan lens

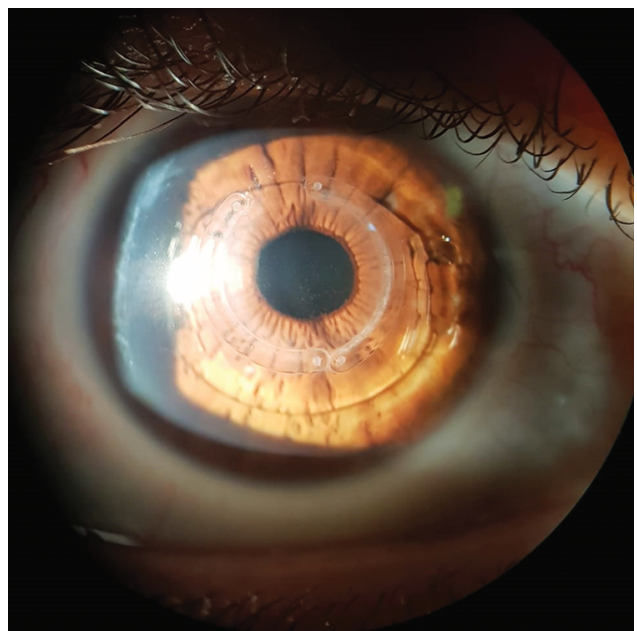

Fig. 1: Patient (MBS) with two Ferrara intracorneal ring segments in association with an Artiflex phakic intraocular lens in OD

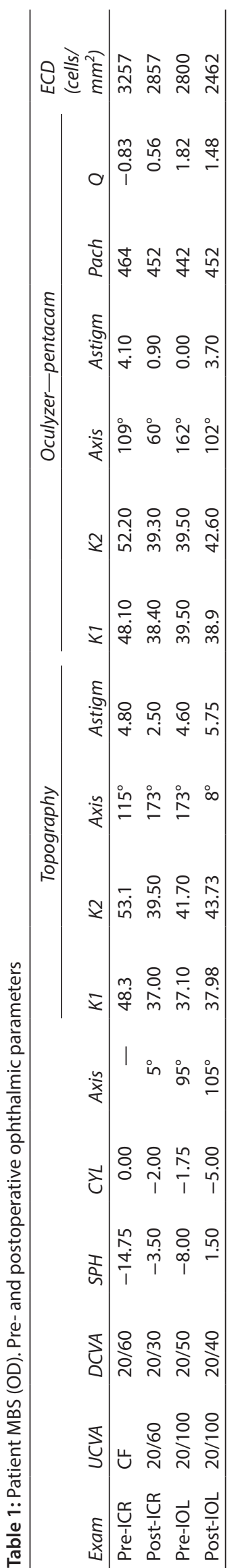


(-19.5 D), improved UCVA, DCVA, and manifest refraction were observed (Table 2).

- Case 3: CESP, 27-year-old male patient. Six months after the implantation of two Ferrara ICRS (160/200) in OD, UCVA and DCVA improved significantly, with a reduction in the manifest refraction and keratometric astigmatism. Topography and tomography showed a significant flattening of the cornea. The patient then received an ICL $(-23 \mathrm{D})$, and six months after implantation, improved UCVA, DCVA, and manifest refraction were observed (Table 3).

- Case 4:VFS, a 32-year-old female patient. After the implantation of two Ferrara ICRS (160/200) in AO, topographic findings, UCVA, and BSCVA improved significantly, and reduction in the manifest refraction and keratometric astigmatism was also observed in AO. Six months after Ferrara implantation, an ICL (-13.00 D) and an Artiflex (-14.5 D) PIOL were implanted in OD and OS, respectively. Six months later, a significant improvement in refraction was found in $\mathrm{AO}$, with improved UCVA and DCVA (Table 4-OD; Table 5-OS).

\section{Discussion}

This case series illustrates the sequential implantation of Ferrara ICRS for the management of keratoconus followed by the implantation of a PIOL to correct residual refractive error. The results demonstrated that this combined procedure was successful in reshaping the cornea and improving the visual acuity of patients.

Several studies have shown that the implantation of Ferrara ICRS for the management of mild to moderate forms of keratoconus is safe and predictable, resulting in improved visual outcomes in all eyes studied. ${ }^{16-18}$ Tunnel creation with femtosecond laser for the implantation of ICRS has also been shown to be minimally invasive, resulting in improved visual acuity. ${ }^{8,13}$ These findings are in agreement with the results in the present study, which showed a significant flattening of the cornea and improved UCVA and DCVA after ICRS implantation, without any intraoperative or postoperative complications.

Although the corneal shape improved after ICRS implantation, patients still presented a significant degree of ametropia, in agreement with previous reports. ${ }^{8,19,20}$ Because the patients were intolerant to contact lenses and the high residual myopia contraindicated the use of laser refractive surgery, 9,10 we opted for the implantation of PIOLs. The approach employed in the present study has been previously termed as "therapeutic bioptics". ${ }^{21}$ It is considered therapeutic because the main goal of the combined procedure is to restore vision, differently from elective refractive surgery, whose aim is to reduce dependence on vision correction (spectacles or contact lenses).

The choice of the PIOL was based on the axis of the corneal astigmatism. When the maximum corneal curvature (K2) was at or near the $180^{\circ}$ axis, ICL was selected. When $\mathrm{K} 2$ was at or near the $90^{\circ}$ axis, Artiflex was chosen if the refractive astigmatism was less than 2.5 D, and Artisan if it was more than 2.5 D. The posterior chamber $\mathrm{ICL}$ is considered to be technically less challenging than anterior chamber iris-fixated Artisan and Artiflex. ${ }^{11}$ Both Artisan and Artiflex follow similar implantation procedures. However, the rigid material of Artisan requires a $5.5 \mathrm{~mm}$ posterior corneal incision, which may induce more postoperative astigmatism. ${ }^{15}$ Artiflex, on the contrary, is made of a foldable material that can be inserted through a smaller incision $(3.2 \mathrm{~mm}$ ) without the need for sutures, reducing induced

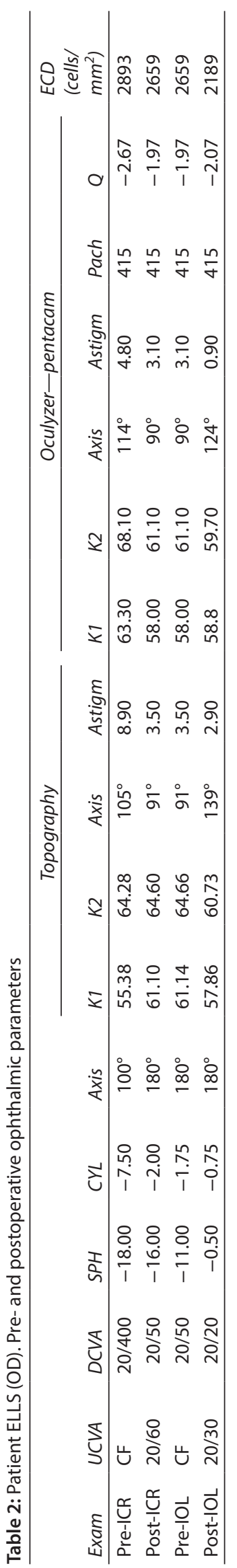



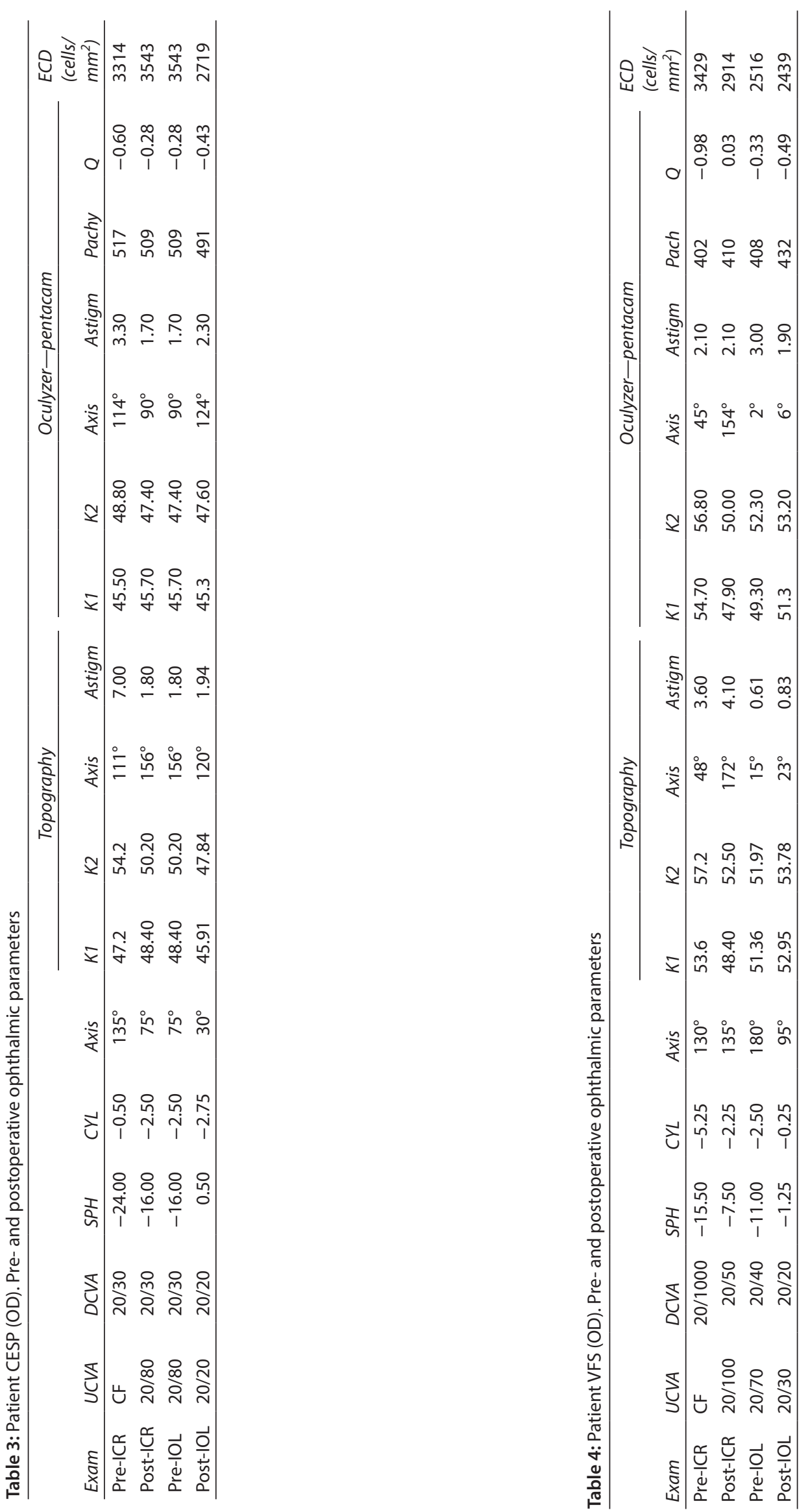


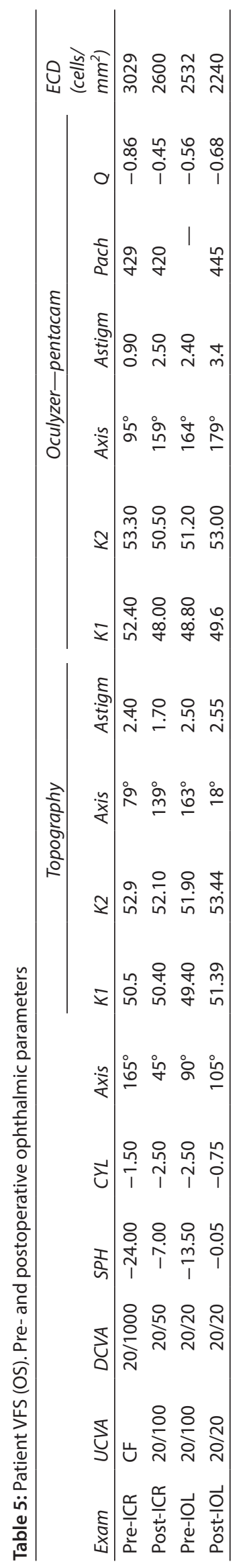

astigmatism. ${ }^{22}$ Despite their differences, all the PIOLs used in the present study demonstrated to be effective and predictable in correcting myopia in the phakic keratoconic eyes.

After PIOL implantation, the UCVA improved significantly as a result of decreased visual distortion caused by high myopia and astigmatism, in agreement with previous studies. ${ }^{8,13,14}$ Both UCVA and DCVA showed a significant improvement, while the manifest refraction showed a significant reduction, with minimal residual myopia and astigmatism. Additionally, both refraction and keratometry were stable 6 months after the combined procedure.

The results demonstrated by the present study indicate that PIOLs (anterior or posterior) can be safely used to correct residual refractive error after the implantation of Ferrara ICRS in eyes with keratoconus and high myopia. Nonetheless, this procedure can only be considered successful if both refraction the progression of keratoconus can be stabilized. Because of the young age of the treated patients, regular follow-ups will be required to ensure that refraction and the shape of the cornea remain stable over time. Another important question regards the possible corneal tissue damages, especially to endothelial cells, caused by PIOLs. The implantation of posterior chamber $\mathrm{PIOL}^{23,24}$ and iris-fixated IOL (iris-claw lens) ${ }^{25,26}$ have been shown to reduce the endothelial cell density (ECD). Of particular concern with anterior chamber PIOLs is the possible cell loss due to mechanical contact between the PIOL and the endothelium of the cornea. ${ }^{22}$ However, several studies demonstrated that Artisan/Artiflex do not touch the endothelium, and ECD is stable in the long-term. ${ }^{27,28}$ A recent study demonstrated that the implantation of Artiflex was predictable for the correction of moderate to high myopia, with no statistically significant changes in EDC or polymegathism after 6 months of follow-up. ${ }^{29}$ Great care must, however, be employed in case selection based on preoperative parameters, such as patient age, iris configuration, and anterior chamber depth of at least $3.2 \mathrm{~mm}$ from the epithelium. ${ }^{30}$

\section{ConClusion}

The results obtained in this small case series demonstrated that ICRS implantation followed by PIOL implantation provides good results with predictability and efficacy when the case is well selected, the correct surgical technique is employed, and postoperative care is provided in the long-term.

\section{Clinical Significance}

The combined procedure presented here is an important alternative that should be considered to provide young patients with improved visual acuity in a moment in their lives when they are at their most productive.

\section{ACKNowledgment}

The authors would like to thank Mr Antonio Carlos Correa for his assistance in reviewing and translating the text into English.

\section{References}

1. Rabinowitz YS. Keratoconus. Surv Ophthalmol 1998;42(4):297-319. DOI: 10.1016/S0039-6257(97)00119-7.

2. Jhanji V, Sharma N, Vajpayee RB. Management of keratoconus: current scenario. Br J Ophthalmol 2011;95(8):1044-1050. DOI: 10.1136/ bjo.2010.185868. 
3. Hamdi IM. Preliminary results of intrastromal corneal ring segment implantation to treat moderate to severe keratoconus. J Cataract Refract Surg 2011;37(6):1125-1132. DOI: 10.1016/j.jcrs.2010.12.048.

4. Lago MA, Ruperez MJ, Monserrat C, et al. Patient-specific simulation of the intrastromal ring segment implantation in corneas with keratoconus. J Mech Behav Biomed Mater 2015;51:260-268. DOI: 10.1016/j.jmbbm.2015.07.023.

5. Chan E, Snibson GR. Current status of corneal collagen cross-linking for keratoconus: a review. Clin Exp Optom 2013;96(2):155-164. DOI: 10.1111/cxo.12020.

6. Viswanathan D, Kumar NL, Males JJ. Outcome of corneal collagen crosslinking for progressive keratoconus in paediatric patients. Biomed Res Int 2014;2014:140461. DOI: 10.1155/2014/140461.

7. Ivarsen A, Hjortdal J. Collagen cross-linking for advanced progressive keratoconus. Cornea 2013;32(7):903-906. DOI: 10.1097/ ICO.0b013e31828321dd.

8. Coskunseven E, Onder M, Kymionis GD, et al. Combined Intacs and posterior chamber toric implantable Collamer lens implantation for keratoconic patients with extreme myopia. Am J Ophthalmol 2007;144:387-389. DOI: 10.1016/j.ajo.2007.05.035.

9. Uçakhan OO, Kanpolat A, Ozdemir O. Contact lens fitting for keratoconus after Intacs placement. Eye Contact Lens 2006;32:75-77. DOI: 10.1097/01.icl.0000174749.96423.ca.

10. Hladun L, Harris M. Contact lens fitting over intrastromal corneal rings in a keratoconic patient. Optometry 2004;75:48-54. DOI: 10.1016/ S1529-1839(04)70010-X.

11. El Danasoury MA, El Maghraby A, Gamali TO. Comparison of iris-fixed Artisan lens implantation with excimer laser in situ keratomileusis in correcting myopia between -9.00 and -19.50 diopters: a randomized study. Ophthalmology 2002;109(5):955-964. DOI: 10.1016/S01616420(02)00964-8.

12. Malecaze FJ, Hulin $\mathrm{H}$, Bierer $\mathrm{P}$, et al. A randomized paired eye comparison of two techniques for treating moderately high myopia: LASIK and artisan phakic lens. Ophthalmology 2002;109(9):1622-1630. DOI: 10.1016/S0161-6420(02)01164-8.

13. Alfonso JF, Lisa C, Fernández-Vega L, et al. Intrastromal corneal ring segments and posterior chamber phakic intraocular lens implantation for keratoconus correction. J Cataract Refract Surg 2011;37(4):706-713. DOI: 10.1016/j.jcrs.2010.10.060.

14. Kamburoğlu G, Ertan A, Bahadir M. Implantation of Artisan toric phakic intraocular lens following Intacs in a patient with keratoconus. J Cataract Refract Surg 2007;33(3):528-530. DOI: 10.1016/ j.jcrs.2006.10.047.

15. Coullet J, Guëll JL, Fournié P, et al. Iris-supported phakic lenses (rigid vs foldable version) for treating moderately high myopia: randomized paired eye comparison. Am J Ophthalmol 2006;142(6):909-916. DOI: 10.1016/j.ajo.2006.07.021.

16. Siganos D, Ferrara P, Chatzinikolas K, et al. Ferrara intrastromal corneal rings for the correction of keratoconus. J Cataract Refract Surg 2002;28(11):1947-1951. DOI: 10.1016/S0886-3350(02)01495-5.
17. Miranda D, Sartori M, Francesconi C, et al. Ferrara Intrastromal Corneal Ring Segments for Severe Keratoconus. J Refract Surg 2003;19(6):645-653.

18. Ameerh MA, Hamad $\mathrm{Gl}, \mathrm{Ababneh} \mathrm{OH}$, et al. Ferrara ring segments implantation for treating keratoconus. Int J Ophthalmol 2012;5(5): 586-590. DOI: 10.3980/j.issn.2222-3959.2012.05.08.

19. Shabayek MH, Alio JL. Intrastromal corneal ring segment implantation by femtosecond laser for keratoconus correction. Ophthalmology 2007;114:1643-1652. DOI: 10.1016/j.ophtha.2006.11.033.

20. Torquetti L, Berbel RF, Ferrara P. Long-term follow-up of intrastromal corneal ring segments in keratoconus. J Cataract Refract Surg 2009;35:1768-1773. DOI: 10.1016/j.jcrs.2009.05.036.

21. Ambrósio Jr R. Intracorneal ring segment, phakic IOL implantation enable keratoconus management without keratoplasty. Ocular Surgery News 2011.

22. Dick HB, Budo C, Malecaze F, et al. Foldable Artiflex phakic intraocular lens for the correction of myopia: two-year follow-up results of a prospective European multicenter study. Ophthalmology 2009;116(4):671-677. DOI: 10.1016/j.ophtha.2008.12.059.

23. Sanders DR, Doney K, Poco M. ICL in Treatment of Myopia Study Group. United States Food and Drug Administration clinical trial of the implantable collamer lens (ICL) for moderate to high myopia: three-year follow-up. Ophthalmology 2004;111(9):1683-1692. DOI: 10.1016/j.ophtha.2004.03.026.

24. Al Sabaani N, Al Assiri A, Al Torbak A, et al. Outcome of posterior chamber phakic intraocular lens procedure to correct myopia. Saudi J Ophthalmol 2013;27(4):259-266. DOI: 10.1016/j.sjopt.2013.06.009.

25. Budo C, Hessloehl JC, Izak M, et al. Multicenter study of the Artisan phakic intraocular lens. J Cataract Refract Surg 2000;26:1163-1171. DOI: 10.1016/S0886-3350(00)00545-9.

26. Benedetti S, Casamenti V, Benedetti M. Long-term endothelial changes in phakic eyes after Artisan intraocular lens implantation to correct myopia: five-year study. J Cataract Refract Surg 2007;33: 784-790. DOI: 10.1016/j.jcrs.2007.01.037.

27. Pop M, Payette Y. Initial results of endothelial cell counts after Artisan lens for phakic eyes: an evaluation of the United States Food and Drug Administration Ophtec study. Ophthalmology 2004;111:309. DOI: 10.1016/j.ophtha.2003.05.025.

28. Tahzib NG, Nuijts RM, Wu WY, et al. Long-term study of Artisan phakic intraocular lens implantation for the correction of moderate to high myopia: ten-year follow-up results. Ophthalmology 2007;114: 1133-1142. DOI: 10.1016/j.ophtha.2006.09.029.

29. Nassiri N, Ghorbanhosseini S, Jafarzadehpur E, et al. Visual acuity, endothelial cell density and polymegathism after iris-fixated lens implantation. Clin Ophthalmol 2018;12:601-605. DOI: 10.2147/OPTH. S157501.

30. Saxena R, Boekhoorn SS, Mulder PG, et al. Long-term follow-up of endothelial cell change after Artisan phakic intraocular lens implantation. Ophthalmology 2008;115(4):608e1-613e1. DOI: 10.1016/ j.ophtha.2007.05.036. 